\title{
Fatores de Risco para Sépsis Associada aos Cuidados de Saúde em Recém-nascidos de Muito Baixo Peso
}

\section{Risk Factors for Healthcare Associated Sepsis in Very Low Birth Weight Infants}

\author{
Helena PEREIRA $\triangle 1,2$, Ema GRILO ${ }^{2,3}$, Patrícia CARDOSO ${ }^{2,3}$, Natália NORONHA ${ }^{2,3}$, Cristina RESENDE ${ }^{3}$ \\ Acta Med Port 2016 Apr;29(4):261-267 • http://dx.doi.org/10.20344/amp.6839
}

\begin{abstract}
RESUMO
Introdução: As infeções associadas aos cuidados de saúde constituem uma importante causa de morbi-mortalidade neonatal, levando a um aumento do tempo de internamento e consequentemente dos seus custos. O objetivo deste estudo foi avaliar a taxa de incidência de infeções associadas aos cuidados de saúde e os seus principais fatores de risco em recém-nascidos de muito baixo peso. Material e Métodos: Estudo retrospetivo dos recém-nascidos de muito baixo peso internados numa maternidade com apoio perinatal diferenciado, durante um período de 10 anos (2005-2014). Foi analisada a existência de associação entre vários fatores de risco e a ocorrência de infeções associadas aos cuidados de saúde.

Resultados: Foram internados 461 recém-nascidos de muito baixo peso. Houve 110 episódios de infeções associadas aos cuidados de saúde em 104 recém-nascidos e 53 episódios de sépsis associada a cateterismo venoso central. A densidade de sépsis foi 7,5/1 000 dias de internamento e a densidade de sépsis associada ao cateterismo venoso central 22,6/1 000 dias de utilização. Os recém-nascidos com infeções associadas aos cuidados de saúde apresentaram uma média de peso ao nascimento e idade gestacional inferior $(959 \pm 228 \mathrm{~g}$ vs $1191 \pm 249 \mathrm{~g})$ e $(27,6 \pm 2$ vs 29,8 $\pm 2,2$ semanas $), p<0,001$. Após ajuste à idade gestacional e peso ao nascimento verificámos associação entre infeções associadas aos cuidados de saúde e antibioterapia em D1, duração de cateterismo venoso central e da nutrição parentérica. Após regressão logística, mantiveram-se como fatores de risco independentes com significância estatística, a idade gestacional e a duração da nutrição parentérica.
\end{abstract}

Discussão: Os fatores de risco independentes para infeções associadas aos cuidados de saúde foram a idade gestacional e a duração da nutrição parentérica.

Conclusão: Por cada semana a mais na idade gestacional o risco de infeções associadas aos cuidados de saúde diminuiu em $20 \%$ e por cada dia de nutrição parentérica o risco aumentou em $22 \%$.

Palavras-chave: Factores de Risco;Infecção Hospitalar; Recém-Nascido de Muito Baixo Peso; Sépsis.

\section{ABSTRACT}

Introduction: Healthcare associated infections in very low birth weight infants are associated with significant morbidity and mortality and are also a cause of increased length of stay and hospital costs. The objective of this study was to evaluate the rate of healthcareassociated sepsis and associated risk factors in very low birth weight infants.

Material and Methods: Retrospective observational study including very low birth weight infants hospitalized in a Neonatal Intensive Care Unit during ten years (2005-2014). We evaluated the association between several risk factors and healthcare-associated sepsis. Results: 461 very low birth weight infants were admitted. There were 110 episodes of HS in 104 very low birth weight infants and 53 episodes of sepsis associated with central vascular catheter. The density of the sepsis was 7.5/1 000 days of hospitalization and the density of central vascular catheter - associated sepsis was 22.6/1 000 days of use. The infants with HS had lower average birth weight and gestational age $(959 \pm 228 \mathrm{~g}$ vs $1191 \pm 249 \mathrm{~g}$ and $27.6 \pm 2$ vs $29.8 \pm 2.2$ weeks $), \mathrm{p}<0.001$. After adjusting for birth weight and gestational age we verified an association between healthcare-associated sepsis and antibiotic therapy in D1, the duration of parenteral nutrition and central vascular catheter. After logistic regression only the gestational age and duration of parenteral nutrition remained as independent significant risk factors for healthcare-associated sepsis.

Discussion: The independent factors for healthcare-associated sepsis are gestational age and duration of parenteral nutrition.

Conclusion: For each extra week on gestational age the risk declined in $20 \%$ and for each day of NP the risk increased $22 \%$.

Keywords: Cross Infection; Infant, Low Birth Weight; Risk Factors; Sepsis.

\section{INTRODUÇÃO}

As infeções associadas aos cuidados de saúde (IACS) constituem um problema grave e temível nas unidades de cuidados intensivos neonatais, estando associadas ao aumento da morbi-mortalidade, do tempo de internamento e dos seus custos..$^{1,2}$

As taxas de IACS neonatais com gravidade associada, como sépsis, reportadas em estudos internacionais variam entre $0,1 \%$ nos recém-nascidos de termo e $21-30 \%$ nos recém-nascidos de muito baixo peso (RNMBP), tendo uma relação inversamente proporcional ao peso ao nascimento. ${ }^{1,3}$

Nos últimos anos, os avanços técnicos e a melhoria da assistência ao nível dos cuidados intensivos neonatais (UCIN) conduziram a um aumento da sobrevida dos grandes prematuros e dos RNMBP. Esta melhoria resulta, muitas vezes, de um maior recurso a procedimentos invasivos (cateterismo venoso central, nutrição parentérica, ventilação mecânica invasiva) e utilização de antibioterapia de

1. Serviço de Pediatria. Centro Hospitalar de Trás-os-Montes e Alto Douro. Unidade de Vila Real. Vila Real. Portugal.

2. Serviço de Pediatria. Maternidade Bissaya Barreto. Centro Hospitalar e Universitário de Coimbra. Coimbra. Portugal.

3. Serviço de Pediatria. Hospital Pediátrico de Coimbra: Centro Hospital e Universitário de Coimbra. Coimbra. Portugal.

$\triangle$ Autor correspondente: Helena Pereira. hiapereira@gmail.com

Recebido: 23 de julho de 2015 - Aceite: 30 de novembro de 2015 | Copyright @ Ordem dos Médicos 2016 
largo espectro, associando-se a maior duração de internamento e prestação de serviço por múltiplos cuidadores. Tais fatores, aliados à imaturidade do sistema imunológico, gastrointestinal e das funções de barreira da pele, facilitam a colonização e invasão por microrganismos potencialmente patogénicos, tornando a sépsis associada aos cuidados de saúde, uma das complicações mais frequentes nas UCIN. ${ }^{4-6}$

Têm sido descritos na literatura vários fatores de risco associados a sépsis, ${ }^{7-9}$ incluindo sépsis associada ao $\mathrm{CVC}^{10,11} \mathrm{e}$ factores de risco para alguns tipos de microrganismos. ${ }^{12-15}$ Muitas das infeções associadas aos CVC são causadas por Staphylococcus coagulase negativos (SCN) ou outros colonizadores da pele circundante à inserção do CVC ou das suas conexões.

A vigilância prospetiva das taxas de incidência de sépsis é considerada um instrumento fundamental de controlo de qualidade. ${ }^{16}$

Desde 2001 que o programa de vigilância epidemiológica de infeção nas UCIN passou a integrar o Programa Nacional de Controlo da Infeção (PNCl), permitindo um melhor conhecimento epidemiológico da realidade a nível nacional.

A monitorização dos fatores de risco individuais das UCIN torna-se preponderante pois permite a implementação de medidas de controlo de infeção mais dirigidas e eficazes.

O presente estudo teve como objetivo avaliar as taxas de incidência de infecções associadas aos cuidados de saúde, nomeadamente a incidência de sépsis, a incidência de sépsis associada a cateter vascular, a incidência de pneumonia associada ao ventilador e identificar fatores de risco associados à ocorrência de IACS nos RNMBP.

\section{MATERIAL E MÉTODOS}

Estudo retrospetivo, observacional, longitudinal e analítico dos RNMBP internados na UCIN de uma maternidade com apoio perinatal diferenciado, durante o período de dez anos (01/01/2005 a 31/12/2014).

Neste estudo foram usados os critérios de infeção definidos pelo Programa Nacional de Controlo de Infeções (PNCl). ${ }^{17}$

Consideramos estar na presença de IACS se os sinais e sintomas tiveram início mais de 72 horas após a admissão.

A sépsis clínica é definida pela presença de dois dos seguintes sinais e sintomas de infeção sistémica: febre (temperatura axilar $>38^{\circ} \mathrm{C}$ ), instabilidade térmica, hipotermia (temperatura axilar $<36,5^{\circ} \mathrm{C}$ ), episódios de apneia (> 20 segundos), episódios de bradicardia ( $\mathrm{FC}<80 / \mathrm{min}$ ), taquicardia (> 200/min), tempo de reperfusão capilar $>$ dois segundos, acidose metabólica (BE $\leq 12 \mathrm{mEq} / \mathrm{l}$ ) sem outra etiologia que o justifique, hiperglicémia (> $140 \mathrm{mg} / \mathrm{dL}$ ) sem justificação, aumento da necessidade de $\mathrm{O}_{2}$, agravamento dos parâmetros ventilatórios, necessidade de reintubação, hipotonia, hiporreatividade, intolerância alimentar e pelo menos um dos seguintes: proteína $\mathrm{C}$ reativa positiva $(>2$ $\mathrm{mg} / \mathrm{dL}$ ), leucocitose/ leucopenia (> $30000 / \mathrm{mm}^{3} /<5000 /$ $\mathrm{mm}^{3}$ ), relação neutrófilos imaturos/totais $>0,2$.

Septicémia é definida como sépsis clínica com hemocultura positiva (colheita assética por punção venosa).

Consideramos sépsis associada ao CVC se a hemocultura periférica e a hemocultura do cateter são ambas positivas para o mesmo agente, com o mesmo antibiograma, em doente com CVC ou com CVC até 48 horas antes do aparecimento dos sinais e sintomas.

A razão de utilização do CVC foi calculada dividindo o número total de dias de utilização do CVC pelo número total de dias de internamento x 100 .

A razão de utilização do TET (tubo endotraqueal) foi calculada dividindo o número total de dias de utilização do TET pelo número total de dias de internamento em cuidados intensivos $\mathrm{x} 100$.

Nas infeções associadas a dispositivo (CVC, TET) foram calculadas as taxas de incidência relacionadas com a exposição ao factor de risco.

As infecções são consideradas como associadas ao respectivo dispositivo invasivo se tiver havido utilização desse dispositivo até 48 horas antes do aparecimento dos sinais e sintomas de infecção. O dispositivo deve ter estado colocado num mínimo de 12 horas para que seja contabilizado um dia de utilização do dispositivo.

A taxa de pneumonia associada ao ventilador foi calculada dividindo o número de pneumonias em doentes ventilados sobre o número total de dias de ventilação x 1000 .

Foi feita a análise descritiva da incidência acumulada de sépsis (número de casos de sépsis por $100 \mathrm{RN}$ em risco), da densidade de incidência de sépsis (número de casos de sépsis por 1000 dias de internamento), da frequência e percentagem de microrganismos identificados e da mortalidade global (número de óbitos nos RN estudados).

Os RN que tiveram sépsis associada aos cuidados de saúde foram denominados RN-IACS, sendo os restantes englobados no grupo RN-NIACS. Foi realizada a análise univariada entre a ocorrência de sépsis e as várias variavéis: idade materna, realização de corticoterapia pré-natal, IG, PN, género, tipo de parto (cesariana ou vaginal), o facto de serem ou não leves para a idade gestacional (LIG), considerando-se LIG os RN cujo PN para a IG foi inferior ao percentil 10 das Tabelas de Fenton, ${ }^{18}$ gravidade da doença inicial avaliada pelo Clinical Risk Index for Babies (CRIB) score, ${ }^{19}$ local de nascimento (foram considerados inborn se nasceram na Maternidade em que foi realizado o presente estudo e outborn se nasceram em outros hospitais), necessidade de reanimação na sala de partos, antibioterapia em D1, idade de início da alimentação entérica, totalidade de dias de internamento e mortalidade. Foram avaliados também a presença ou não de fatores de risco/procedimentos invasivos e a sua duração, nomeadamente ventilação mecânica convencional (VMC), ventilação não invasiva (VNI), cateter venoso central e nutrição parentérica. Nos RN - IACS a ventilação e a nutrição parentérica apenas foram consideradas fatores de risco se as mesmas estavam presentes ao momento do diagnóstico, sendo excluídos os casos em que estes procedimentos foram necessários no 
contexto de quadro séptico.

A análise estatística foi feita utilizando o SPSS versão 20. Foi efetuada a análise bivariada usando o teste de Mann-Whitney para as variáveis quantitativas e o teste de Qui-quadrado/ teste Fisher/ Qui-quadrado de tendência linear (segundo as regras de Cochran) para as variáveis categóricas. Calculou-se os odds ratio $(O R)$ e os respetivos $95 \%$ confidence interval $(95 \% \mathrm{Cl})$. Foi feito o ajuste destes fatores de risco à idade gestacional e ao peso de nascimento $(\mathrm{ORa})$. Para investigar e determinar o efeito de fatores de risco, foi efetuada uma análise de regressão logística para todas as variáveis, considerando-se na análise bivariada, diferença estatística com uma probabilidade de ocorrência inferior a 0,1 . Devido à colinearidade entre a IG e o $P N$, na regressão logística, apenas foi introduzida no modelo a IG. Foi considerada significância estatística se $p<0,05$.

A curva ROC (receiver operating characteristic) foi utilizada para definir os melhores pontos entre os tempos de NP e CVC para a ocorrência de sépsis associada aos cuidados de saúde.

\section{RESULTADOS}

Durante o período em estudo (2005-2014), foram internados na UCIN 461 RN com PN inferior a $1500 \mathrm{~g}$, correspondendo a $17,3 \%(461 / 2655)$ do nosso universo de internamentos na UCIN.

A média da IG foi de 29,3 semanas ( $d p \pm 2,4)$ e o peso médio de $1139 \mathrm{~g}$ ( $\mathrm{dp} \pm 263 \mathrm{~g}$ ); o peso mínimo foi de $440 \mathrm{~g} \mathrm{e}$ o peso máximo de $1490 \mathrm{~g}$. A razão masculino:feminino foi de 1,25:1.

Do total de RNMBP, 127 (27,5\%) eram LIG, 64 (13,9\%) eram RN outborn e o parto ocorreu por cesariana em 317 $(68,7 \%)$.

O valor de CRIB médio foi de 2,48 ( $\mathrm{dp} \pm 2,89$ ).

Do total de RNMBP, 104 (22,5\%) RN tiveram sépsis associada aos cuidados de saúde (RN - IACS $=104)$, correspondendo a 110 episódios de sépsis, dado que um dos RN teve três episódios de sepsis e em quatro RN verificou-se a presença de dois episódios de sepsis. Os RN com mais de uma infecção eram todos $\mathrm{RN}$ extremo baixo peso, ventilados, com NPT e CVC.

A mediana (percentil 10 e percentil 90) do dia de diagnóstico de sépsis foi 9,1 dias (5,3-25,8 dias).

Os 461 RN contabilizaram no total 14572 dias de internamento, com uma média de 31,6 dias $\pm 25,7$ dias. O número total de dias de CVC foi de 2345 dias.

No período analisado faleceram $20 \mathrm{RN}(4,3 \%)$.

$\mathrm{Na}$ Tabela 1 registam-se as características clinicas e demográficas da amostra.

Nos RNMBP a densidade de incidência de sépsis foi de 7,5 por 1000 dias de internamento (110/14 572). A densidade de incidência de sépsis associada ao CVC foi de 22,6 episódios por 1000 dias de utilização do CVC (53/2 345).

A Tabela 2 explicita a incidência, densidade de incidência da sépsis e da sépsis associado ao CVC dos RN em estudo para os RNMBP e RN extremo baixo peso $(<1000 \mathrm{~g})$. Comparativamente com os RNMBP, os RN extremo baixo peso evidenciam maior taxa de sepsis e maior densidade

Tabela 1 - Características clinicas e demográficas da amostra

\begin{tabular}{|c|c|}
\hline Idade materna média/dp & $30,2 \pm 5,7$ anos \\
\hline Corticoides pré-natais; $\mathrm{n}(\%)$ & $344(90 \%)$ \\
\hline IG média/dp & $29,3 \pm 2,4 \mathrm{~S}$ \\
\hline PN média/dp & $1139 \pm 263 \mathrm{~g}$ \\
\hline Género masculino; n (\%) & $260(56 \%)$ \\
\hline CRIB média/dp & $2,48 \pm 2,89$ \\
\hline LIG; n (\%) & $127(27,5 \%)$ \\
\hline Outborn; n (\%) & $64(13,9 \%)$ \\
\hline Cesariana; n (\%) & $317(68,7 \%)$ \\
\hline $\begin{array}{l}\text { Dias de internamento média/dp } \\
\text { Total de dias de internamento }\end{array}$ & $\begin{array}{l}31,6 \pm 25,7 d \\
14572 d\end{array}$ \\
\hline $\begin{array}{l}\mathrm{N}^{\circ} \mathrm{RN} \text { com CVC (\%) } \\
\text { Total de dias de CVC }\end{array}$ & $\begin{array}{l}254(55 \%) \\
2345\end{array}$ \\
\hline Taxa de IACS-CVC /1 000 dias de utilização & 22,6 \\
\hline Duração da VMC media/dp & $11,9 \pm 19$ \\
\hline $\begin{array}{l}\mathrm{N}^{\circ} \mathrm{RN} \text { com TET }(\%) \\
\text { Total de dias de TET }\end{array}$ & $\begin{array}{l}237(51 \%) \\
2252\end{array}$ \\
\hline Taxa de pneumonia-TET /1 000 dias de utilização & 2,8 \\
\hline Duração média da NP média/dp & $5,0 \pm 4,6$ \\
\hline Mortalidade; n (\%) & $20(4,3 \%)$ \\
\hline
\end{tabular}

IG: idade gestacional; PN: peso nascimento; CRIB: Clinical Risk Index for Babies; LIG: leve para a idade gestacional; dp: desvio padrão; RN: recém-nascido; CVC: cateter vascular central; VMC: ventilação mecânica convencional; NP: nutrição parentérica; TET: tubo endotraqueal. 
Tabela 2 - Taxa de incidências e densidade de incidências da sépsis nosocomial

\begin{tabular}{lcc}
\hline & RNMBP & RNEBP \\
\hline Taxa de sépsis & $23 \%$ & $44 \%$ \\
Taxa de sépsis / hemocultura + & $16 \%$ & $33 \%$ \\
Densidade de incidência de sépsis & 7,5 & 11,2 \\
Densidade de incidência de sépsis hemocultura + & 5,8 & 7,4 \\
Densidade de incidência de sépsis associada a CVC & 22,6 & 23,7 \\
Densidade de incidência de sépsis associada a CVC com hemocultura positiva & 15,6 & 19,1 \\
\hline
\end{tabular}

CVC: cateter vascular central; RNMBP: recém-nascido de muito baixo peso; RNEBP: recém-nascido de extremo baixo peso.

de incidencia de sepsis. Verificou-se positividade de culturas em 73 episódios de sépsis $(66,4 \%)$, correspondendo ao isolamento de 79 espécies de microrganismos.

Os agentes mais isolados foram: Staphylococcus coagulase negativos (47/73), seguido de Staphylococcus aureus (12/73), dos quais quatro eram $S$. aureus meticilino-resistentes, Escherichia coli (7/73) e Candida spp (6/73). Não foram identificadas bacilos gram negativos produtores de ß-lactamases de espectro expandido. Os dados referentes aos microrganismos isolados estão representados na Tabela 3.

Os resultados da análise univariada de fatores de risco para ocorrência de IACS estão representados na Tabela 4. A idade média de início da alimentação entérica foi de 3,0 \pm 1,2 dias nos RN - IACS e de 2,1 $\pm 1,2$ dias nos RN - NIACS.

Após ajuste para a IG e PN, os fatores de risco estatisticamente significativos para IACS foram: início de antibioterapia em D1 (ORa $=1,828,95 \% \mathrm{Cl}=1,049-3,185$. $p=0,033)$, o número de dias de NP $(O R a=1,226,95 \% \mathrm{Cl}$ $=1,139-1,320 . p<0,001)$, o atraso na introdução da alimentação entérica $(O R a=1,285,95 \% \mathrm{Cl}=1,061-1,551$. $p=0,01)$ e o número total de dias de CVC $(O R a=1,047$, $95 \% \mathrm{Cl}=1,002-1,094 \cdot p=0,042$ ).

Após regressão logística identificaram-se como fatores de risco independentes para IACS a idade gestacional (OR $0,80 ; 95 \% \mathrm{Cl} 0,681-0,938 . p=0,006)$ e a duração da NP

Tabela 3 - Microrganismos isolados em hemocultura e mortalidade associada

\begin{tabular}{|ccc|}
\hline Microrganismo & $\mathrm{n}$ & Óbitos $(\mathrm{n}=20)$ \\
\hline Gram + & 63 & $4(20 \%)$ \\
\hline SCN & 47 & 2 \\
Staphylococcus aureus & 12 & 1 \\
Enterococcus spp & 4 & 1 \\
\hline Gram - & 10 & $4(20 \%)$ \\
\hline Escherichia coli & 7 & 3 \\
Klebsiella spp & 1 & 0 \\
Pseudomonas spp & 1 & 0 \\
Enterobacter spp & 1 & 1 \\
\hline Candida spp & 6 & $2(10 \%)$ \\
\hline TOTAL & 79 & $10(50 \%)$ \\
\hline SCN: Staphylococcus coagulase negativo & &
\end{tabular}

(OR 1,22; 95\% Cl 1,122 - 1,332. $p<0,0001)$.

Foi determinado o ponto de coorte (ponto de Youden) a partir do qual os RN apresentam risco estatisticamente significativo de IACS. Verificamos este ponto quando a duração da NP ou do CVC é superior ou igual a 6,5 dias (AUC $=0,82$ e AUC $=0,73$ respetivamente, $p<0,0001$ ) (Figs. 1 e 2).

\section{DISCUSSÃO}

A taxa de incidência de sépsis associada aos cuidados de saúde obtida no nosso estudo, foi inferior ao descrito na literatura e no registo de IACS do Programa Nacional de Controlo de Infeções das UCIN - 7,5/1 000 vs 9/1 000..17 Apesar do peso médio e idade gestacional média serem semelhantes, mantemos taxas de incidência tendencialmente mais baixas.

Os RN - IACS tinham peso e idade gestacional média inferiores aos RN - NIACS. Sendo os nossos dois grupos estatisticamente diferentes, foi efetuada para todos os fatores de risco o ajuste à IG e PN. No nosso estudo e em concordância com outros estudos uni e multicêntricos publicados, valores baixos de PN e de IG associaram-se a maior risco de IACS. ${ }^{7,20-28}$ Por cada semana a mais de idade gestacional, o risco de IACS diminui $20 \%$. RN com IG inferior a 28 semanas tiveram um OR de 5,4 (95\% IC 3,1 - 9,4. $p<0,001)$ e com PN inferior a $1000 \mathrm{~g}$ um OR de $5,2(95 \%$ IC $3,1-8,8 . p<0,001$ ).

Alguns estudos têm sugerido o sexo masculino como fator de risco, ${ }^{28} \mathrm{o}$ que se verificou no nosso estudo, embora sem significado estatístico.

Os RN com scores de gravidade mais elevados (CRIB $\geq 5$ ) associaram-se a maior taxa de IACS durante o internamento, dado corroborado por outros estudos publicados. ${ }^{26}$

A NP tem sido referida como um dos principais fatores de risco para IACS, , 10,22,25,26 o que foi confirmado neste estudo. Por cada dia a mais de NP, o risco de IACS aumenta $22 \%$. Esta associação verificada na nossa unidade reforça a importância da otimização de medidas de boas práticas clínicas e de higienização na preparação, manipulação e administração da mesma.

A introdução precoce de alimentação entérica constitui uma medida protetora de IACS, pois está associada a uma maturação do intestino mais rápida, com diminuição da permeabilidade intestinal e consequente diminuição da translocação bacteriana, para além da invariável diminuição do tempo de duração de nutrição parentérica. 5,21,22,29,30 
Tabela 4 - Fatores de risco para IACS

\begin{tabular}{|c|c|c|c|c|c|}
\hline & $\begin{array}{c}\text { RN-IACS } \\
\mathrm{n}=104\end{array}$ & $\begin{array}{c}\text { RN-NIACS } \\
\mathrm{n}=357\end{array}$ & $\begin{array}{c}\text { OR } \\
(95 \% \text { IC) }\end{array}$ & $\begin{array}{c}\text { ORa } \\
(95 \% \text { IC) }\end{array}$ & $p$ \\
\hline IG média/dp & $27,6 \pm 2 S$ & $29,8 \pm 2,2 \mathrm{~S}$ & & & $<0,001$ \\
\hline PN média/dp & $959 \pm 228 \mathrm{~g}$ & $1191 \pm 249 \mathrm{~g}$ & & & $<0,001$ \\
\hline Género masculino, n (\%) & $36(34,6 \%)$ & $110(30,8 \%)$ & & & ns \\
\hline LIG, $n^{\circ}(\%)$ & $20(19,2 \%)$ & $107(30 \%)$ & $0,5(0,3-0,9)$ & ns & 0,031 \\
\hline CRIB $>4$, média e dp & $4,3 \pm 3,3$ & $1,9 \pm 2,4$ & & ns & $<0,001$ \\
\hline Outborn, n (\%) & $23(22 \%)$ & $41(11,5 \%)$ & $2,1(1,2-3,8)$ & ns & 0,006 \\
\hline Reanimação, n (\%) & $73(70 \%)$ & $177(49,7 \%)$ & $2,3(1,4-3,8)$ & ns & $<0,001$ \\
\hline Antibioterapia D1, n (\%) & $78(75 \%)$ & $155(43,9 \%)$ & $3,8(2,3-6,2)$ & $1,8(1,1-3,6)$ & $<0,001$ \\
\hline VMC, n (\%) & $72(69 \%)$ & $133(37 \%)$ & $3,7(2,3-6)$ & ns & $<0,001$ \\
\hline Dias VMC (média e dp) & $11,9 \pm 19$ & $2,9 \pm 9,7$ & & ns & $<0,001$ \\
\hline VNI, n (\%) & $93(89,4 \%)$ & $201(56 \%)$ & $6,5(3,3-12,6)$ & ns & $<0,001$ \\
\hline Dias VNI, (média e dp) & $19,5 \pm 18$ & $5,9 \pm 11$ & & ns & $<0,001$ \\
\hline CVC $>6$ dias, $n(\%)$ & $68(80 \%)$ & $128(43 \%)$ & $5,3(2,9-9,5)$ & $2,1(1,1-4,1)$ & $<0,001$ \\
\hline Dias CVC (média e dp) & $8,8 \pm 6,7$ & $4 \pm 5,6$ & & $1,1(1,05-1,2)$ & $<0,001$ \\
\hline NP > 6dias, n (\%) & 99 (95\%) & $218(61,1 \%)$ & $12,6(5-31)$ & & $<0,001$ \\
\hline Dias NP (média e dp) & $9,1 \pm 4,5$ & $3,8 \pm 4,1$ & & $1,2(1,1-1,3)$ & $<0,001$ \\
\hline Dia início da AE (média e dp) & $3,0 \pm 1,2$ & $2,1 \pm 1,2$ & & $1,3(1,1-1,6)$ & $<0,001$ \\
\hline Dia AET (média e dp) & 10,9 & 6,1 & & & $<0,001$ \\
\hline Leite materno & $91(88 \%)$ & $328(93 \%)$ & & & ns \\
\hline
\end{tabular}

RN-IACS: recém-nascido com infeção associado aos cuidados de saúde; RN-NIACS: recém-nascido sem infecção associada aos cuidados de saúde; dp: desvio padrão; IG: idade gestacional; PN: peso nascimento; LIG: leve para a IG; CRIB: Clinical Risk Index for Babies; VMC: ventilação mecânica convencional; VNI: ventilação não invasiva; CVC: cateter vascular central; NP: nutrição parentérica; AE: alimentação entérica; AET: alimentaçao enterica total; OR: odds ratio; ORa: ajustada à IG e PN; ns: não significativo.

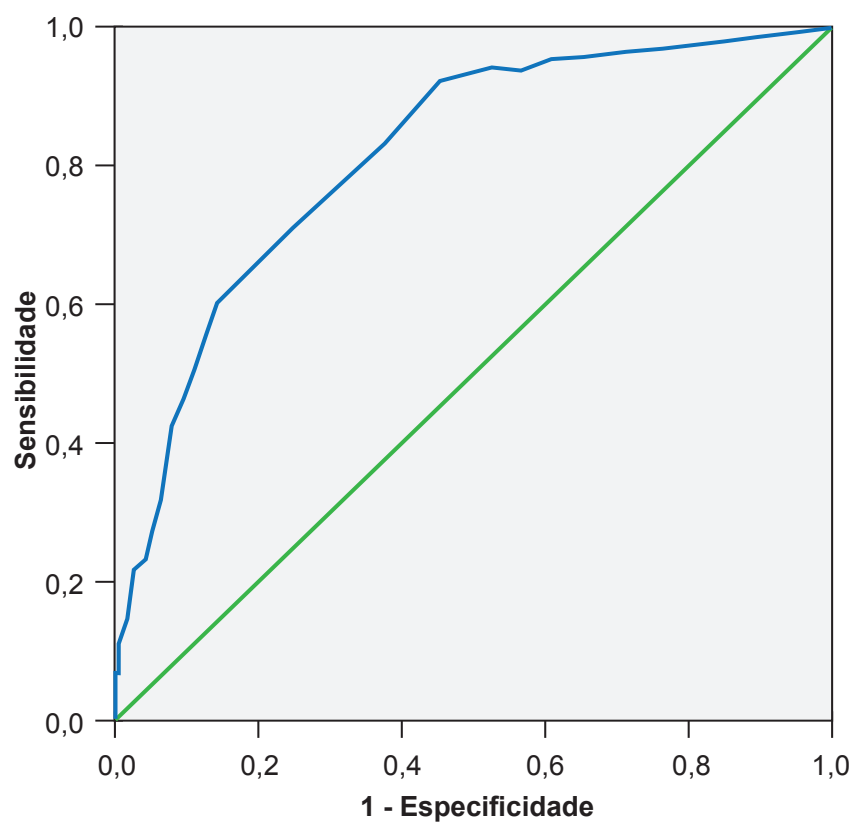

Os segmentos diagonais são elaborados por laços.

Figura 1 - Ponto de Youden entre a duração de NP e a ocorrência de sépsis associada aos cuidados de saúde: 6,5 dias. Com uma capacidade discriminativa (área sob a curva ROC de $A \cup C=\mathbf{0 , 8 1 5}$; IC95\% 0,77-0,861).

NP: nutrição parentérica; ROC: receiver operating characteristic; AUC: area under curve

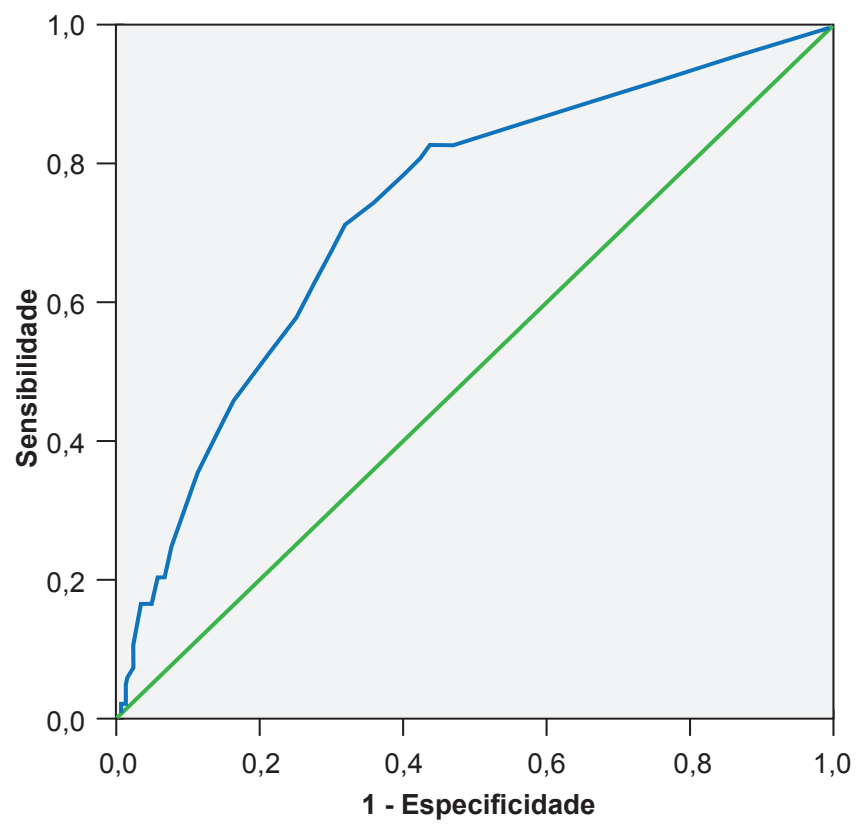

Os segmentos diagonais são elaborados por laços.

Figura 2 - Ponto de Youden entre a duração de CVC e a ocorrência de sépsis associada aos cuidados de saúde: 6,5 dias. Com uma capacidade discriminativa (área sob a curva ROC de AUC = $\mathbf{0 , 7 3 4}$; IC95\% 0,679 - 0,788)

CVC: Cateter vascular central; ROC: receiver operating characteristic; AUC: area under curve 
No nosso estudo, os RN-IACS iniciaram a alimentação entérica mais tarde que os RN-NIACS. Após ajuste para a IG e PN, por cada dia a mais na introdução de alimentação entérica o risco de IACS aumenta $28,5 \%$.

$\mathrm{O}$ aleitamento materno tem sido considerado benéfico na diminuição da incidência de sépsis nosocomial. ${ }^{5,21,22,30,31}$ No nosso estudo não encontramos associação estatisticamente significativa, embora o $p$ fosse 0,006 ,o que pensamos que se deva à elevada taxa de utilização de leite materno em ambos os grupos.

Foi possível estabelecer uma relação estatisticamente significativa entre a presença de CVC e IACS, tal como descrito em vários estudos nacionais e internacionais. 14,20,22,25-28 Esta associação poderá estar relacionada com contaminação durante a inserção, manipulação excessiva, interrupção na manutenção do sistema fechado para administração de antibioterapia ou hemoderivados, utilização de solutos contaminados ou contaminação da pele adjacente ao local de inserção. ${ }^{32}$ Estudos comprovam que após 15 dias de permanência de CVC, o risco de sépsis aumenta drasticamente. ${ }^{30} \mathrm{Na}$ nossa casuística, verificamos que cada dia a mais de CVC acarreta um risco 4,7\% maior de IACS, sendo esse risco estatisticamente significativo quando a duração de CVC é igual ou superior a 6,5 dias.

$\mathrm{O}$ uso de antibioterapia de largo espectro nos RN tem sido também descrito como fator de risco para as IACS, ${ }^{33}$ podendo causar alterações na flora do RN e/ou na flora ambiental, que podem levar ao aparecimento de estirpes multirresistentes ${ }^{32}$ No nosso estudo a utilização de antibioterapia em D1 associou-se a um maior risco de sépsis.

Para além dos fatores de risco intrínsecos e extrínsecos, há também fatores de risco ambiental que podem contribuir para sépsis associada aos cuidados de saúde, nomeadamente a superlotação nas unidades, o número de prestadores de cuidados, a má adesão à higienização das mãos ou aos cuidados de desinfeção na inserção e manutenção/manuseamento dos cateteres, parâmetros não avaliados neste estudo. ${ }^{33}$

A prevenção de IACS baseia-se em estratégias que visam diminuir o risco de infeção através da evicção da transmissão dos microrganismos pelos profissionais de saúde (higiene das mãos e cuidados rigorosos de assepsia na colocação e manutenção de CVC), uso criterioso dos antibióticos, promoção do aleitamento materno e introdução de alimentação enteral precoce. ${ }^{17}$

A prevenção de nascimentos prematuros seria uma das estratégias mais efetivas, mas extremamente difícil de concretizar, pela sua complexidade envolvendo problemas de ordem médica, educativa e social.

A vigilância epidemiológica nas UCIN é fundamental, não só porque permite conhecer a realidade epidemiológica específica das mesmas, mas também pelo facto de os resultados poderem ajudar os profissionais de saúde a direcionar as suas práticas no sentido de melhorar a segurança clínica dos recém-nascidos e a qualidade dos cuidados prestados.

\section{CONCLUSÕES}

Com este trabalho concluímos que a densidade de incidência da sépsis hospitalar no nosso estudo é inferior ao registo nacional.

Os fatores de risco associado às IACS com significância estatística foram a duração da NP e a IG. Por cada semana a mais de IG o risco de infeção diminui $20 \%$, e por cada dia a mais de nutrição parentérica o risco aumentou $22 \%$.

Após determinação do ponto de coorte (ponto de Youden), verificou-se um risco estatisticamente significativo de IACS quando a duração da NP ou do CVC é superior ou igual a 6,5 dias.

A intensificação de boas práticas na prevenção de IACS, incluindo reforço nos cuidados de assepsia, início precoce de alimentação enteral e minimização da utilização prolongada ou inadequada de antibioterapia de largo espectro, deve constituir um desafio diário. A formação contínua dos profissionais de saúde, de modo a aumentar a adesão às medidas instituídas, deve ser uma estratégia a adotar.

\section{PROTECÇÃO DE PESSOAS E ANIMAIS}

Os autores declaram que os procedimentos seguidos estavam de acordo com os regulamentos estabelecidos pelos responsáveis da Comissão de Investigação Clínica e Ética e de acordo com a Declaração de Helsínquia da Associação Médica Mundial.

\section{CONFIDENCIALIDADE DOS DADOS}

Os autores declaram ter seguido os protocolos do seu centro de trabalho acerca da publicação de dados.

\section{CONFLITOS DE INTERESSE}

Os autores declaram não terem qualquer conflito de interesse relativamente ao presente artigo.

\section{FONTES DE FINANCIAMENTO}

Os autores declaram não ter recebido subsídios ou bolsas para a elaboração do artigo.

\section{REFERÊNCIAS}

1. Romanelli RM, Anchieta LM, Carvalho EA, Silva LF, Nunes R, Mourão $P$ et al. Risk factors for laboratory confirmed bloodstream infection in neonates undergoing surgical procedures. Braz J Infect Dis. 2014;18:400-5

2. Sadowska-Krawczenko I, Jankowska A, Kurylak A. Healthcare- associated infections in a neonatal intensive care unit. Arch Med Sci. 2012;8:854-64.

3. Pinho L, Pinto J, Braga AC, Gouveia S, Matos L, Pombeiro J et al. Infeções associadas aos cuidados de saúde numa Unidade de Cuidados Intensivos Neonatais: avaliação da eficácia das estratégias 
de prevenção implementadas. Nascer Crescer. 2013;22:210-5.

4. Polin RA, Denson S, Brady MT and the Committee on Fetus and Newborn and Committee on Infectious Diseases. Epidemiology and diagnosis of health care -associated infectious in the NICU. Pediatrics. 2012;e1104-8.

5. Polin RA, Denson S, Brady MT and the Committee on Fetus and Newborn and Committee on Infectious Diseases. Strategies for prevention of health care -associated infectious in the NICU. Pediatrics. 2012;129:e1085-91.

6. Kamath S, Mallaya S, Shenoy S. Nosocomial infections in neonatal intensive care units: profile, risk factor assessment and antibiogram. Indian J Pediatr. 2010;77:37-9.

7. Holmes A, Doré CJ, Saraswatula A, Bamford KB, Richards MS, Coello $\mathrm{R}$, et al. Risk factors and recommendations for rate stratification for surveillance of neonatal healthcare bloodstream infection. J Hosp Infect. 2003;68:66-72.

8. Nagata E, Brito AS, Matsuo T. Nosocomial infections in neonatal intensive care unit patient: inicidence and risk factors. Am J Infect Control. 2002;30:26-31.

9. Kawagoe JY, Segre CA, Pereira CR, Cardoso MF, Silva CV, Fukushima JT. Risk factors for nosocomial infection in critically ill newborns: a 5-years prospective cohort study. Am J Infect Control. 2001;29:109-14.

10. Van der Zwet WC, Kaiser AM, van Elburg RM, Berkhof J, Fetter WP, Parlevliet GA, et al. Nosocomial infections in a Dutch neonatal intensive care unit: surveillance study with definitions for infection specifically adapted for neonates. J Hosp Infect. 2005;61:300-11.

11. Hsu JF, Tsai MH, Huang HR, Lien R, Chu SM, Huang CB. Risk factors of catheter-related bloodstream infection with percutaneously inserted central venous catheters in very low birth weight infants: a center's experience in Taiwan. Pediatr Neonatol. 2010;51:336-42.

12. Crivaro V, Bogdanović L, Bagattini M, Lula VD, Catania M, Raimondi $F$ et al. Surveillance of healthcare-associated infections in a neonatal intensive care unit in Italy during 2006-2010. BMC Infect Dis. 2015;15:152.

13. Graham PL. Staphylococcal and enterococcal infections in the neonatal intensive care unit. Semin Perinatol. 2002;26:322-31.

14. Graham PL 3rd, Begg MD, Larson E, Della-Latta P, Allen A, Saiman L. Risk Factors for late onset gram negative sepsis in low birth weight infants hospitalized in the neonatal intensive care unit. Pediatr Infect Dis J. 2006;25:113-7.

15. Feja KN, Wu F, Roberts K, Loughrey M, Nesin M, Larson E et al. Risk factors for candidemia in critically ill infants: a matched case control study. J Pediatr. 2005;147:156-61.

16. Neto MT, Serelha M. Vigilância prospetiva da infeção relacionada com a prestação de cuidados de saúde numa Unidade de Cuidados Intensivos Neonatais - uma experiência de seis anos. Acta Pediatr Port. 2009;40:150-3.

17. Protocolo para a Vigilância Epidemiológica das infeções nosocomiais nas Unidades de Cuidados Intensivos Neonatais. Direção Geral da
Saúde, 2007.

18. Fenton TR. A new growth chart for preterm babies: Babson and Benda's chart update with recent data and new format. BMC Pediatr. 2003;3-13.

19. International Neonatal Network. The CRIB (clinical risk index for babies) score: a tool for assessing initial neonatal risk and comparing performance of neonatal intensive care units. Lancet. 1993;342:193-8.

20. Stoll BJ, Hansen N, Fanaroff, Wright LL, Carlo WA, Ehrenkranz RA et al. Late onset sepsis in VLBW neonates: the experience of the NICHD. Pediatrics. 2002;110:285-91.

21. Ronnestad A, Abrahamsen TG, Medbo S, Reigstad H, Lossius K, Kaaresen PI, et al. Late onset septicemia in Norwegian national cohort of extremely premature infants receiving very early full human milk feeding. Pediatrics. 2005;115:E269-76.

22. Downey LC, Smith PB, Benjamin DK. Risk factors and prevention of late onset sepsis in premature infants. Early Hum Dev. 2010;86:S7-12.

23. Hornik CP, Fort P, Clark RH, Watt K, Benjamin DK, Smith PB, et al. Early and late onset sepsis in very low birth weight infants from a large group of Neonatal Intensive Care Unit. Early Hum Dev. 2012;88:S69-74.

24. Curtis C; Shetty N. Recent trends and prevention of infection in the neonatal intensive care unit. Curr Opin Infect Dis. 2008;21:350-6.

25. Auriti C, Maccallini A, Liso GD, Ciommo VD, Ronchetti MP, Orzalesi M Risk factors for nosocomial infection in the neonatal intensive care unit. J Hosp Infect. 2003:53:25-30.

26. Couto RC, Pedrosa TM, Tofani CP, Pedrosa TM. Risk factors for nosocomial infection in a neonatal intensive care unit. Infect Control Hosp Epidemiol. 2006;27:571-5.

27. Babazono A, Kitajima H, Nishimaki S, Nakamura T, Shiga A, Hayakawa $M$, et al. Risk factors for nosocomial infection in the neonatal intensive care unit by the Japanese Nosocomial Infection Surveillance (JANIS). Acta Med Okayama. 2008;62:261-8.

28. Vergnano S, Menson E, Kennea N, Embleton N, Bedford R, Watts T, et al. Neonatal infection in England: the NeonIN surveillance network. Arch Dis Child Fetal Neonatal. 2011;96:F9-14.

29. Manzoni P, De Luca D, Stronati M, Jacqz-Aigrain E, Ruffinazzi G, Luparia $M$, et al. Prevention of Nosocomial Infections in Neonatal Intensive Care Units. Am J Perinatol. 2013;30:81-8.

30. De Silva A, Jones PW, Spencer SA. Does human milk reduce infection rates in preterm infants? A systematic review. Arch Dis Child Fetal Neonatal Ed. 2004;89:F509-13.

31. Bentlin MR, Rugolo L, Ferrari L, on behalf of the Brazilian Neonatal Research Network. Practices related to late-onset sepsis in very low birth weight preterm infants. J Pediatr. 2015;91:168-74.

32. Saiman L. Strategies for prevention of nosocomial sepsis in the neonatal intensive care unit. Curr Opin Pediatr. 2006;18:101-6.

33. Verstraete E, Boelens J, De Coen K, Claeys G, Vogelaers D, Vanhaesebrouck $P$, et al. Healthcare-associated bloodstream infections in a neonatal intensive care unit over a 20-year period (1992-2011): trends in incidence, pathogens, and mortality. Infect Control Hosp Epidemiol. 2014;35:511-8. 